Research Paper

\title{
Clinical Significance of CD163+ and CD68+ Tumor-associated Macrophages in High-risk HPV-related Cervical Cancer
}

\author{
Xiao-Jing Chen ${ }^{1 *}$, Ling-Fei Han ${ }^{4^{*}}$, Xiang-Guang $\mathrm{Wu}^{{ }^{*}}$, Wen-Fei Wei ${ }^{1}$, Lan-Fang $\mathrm{Wu}^{5}$, Hong-Yan $\mathrm{Yi}^{1}$, \\ Rui-Ming Yan ${ }^{1}$, Xiang-yang Bai ${ }^{1}$, Mei Zhong ${ }^{1}$, Yan-hong Y ${ }^{1}{ }^{1}$, Li Liang ${ }^{3 凶}$, Wei Wang1,2®
}

1. Department of Obstetrics and Gynecology, Nanfang Hospital, Southern Medical University/The First School of Clinical Medicine, Southern Medical University, Guangzhou, Guangdong Province, People's Republic of China

2. Department of Obstetrics and Gynecology, the First Affiliated Hospital, Guangzhou Medical University, Guangzhou, Guangdong Province, People's Republic of China

3. Department of Pathology, Nanfang Hospital, Southern Medical University/The First School of Clinical Medicine, Southern Medical University, Guangzhou, Guangdong Province, People's Republic of China

4. Department of Minimally Invasive Gynecologic Surgery, Shanghai First Maternity and Infant Hospital, Tongji University School of Medicine, Shanghai, People's Republic of China

5. Department of Obstetrics and Gynecology, the Third Affiliated Hospital, Southern Medical University, Guangzhou, Guangdong Province, People's Republic of China

* Xiao-Jing Chen, Ling-Fei Han, and Xiang-Guang Wu contributed equally to this work.

$\bowtie$ Corresponding authors: Wei Wang, E-mail: smugowwang@126.com, Tel: 86-20-83062652; Li Liang, E-mail: 1li@fimmu.com, Tel: 86-20-61648223

(c) Ivyspring International Publisher. This is an open access article distributed under the terms of the Creative Commons Attribution (CC BY-NC) license (https://creativecommons.org/licenses/by-nc/4.0/). See http://ivyspring.com/terms for full terms and conditions.

Received: 2017.06.13; Accepted: 2017.08.16; Published: 2017.10.17

\begin{abstract}
Objective. To explore the influence of M2-polarized tumor-associated macrophages (TAMs) on high-risk human papillomavirus (hr-HPV)-related cervical carcinogenesis and metastasis.

Methods. CD68+ and CD163+ macrophages were examined immunohistochemically in a series of 130 samples, including 26 cases of normal cervical tissues, 59 cases of cervical intraepithelial neoplasia ( $\mathrm{CIN}$ ), and 45 cases of squamous cell carcinoma (SCC), and the results were statistically analyzed. The macrophage count was corrected for the epithelial and stromal compartments respectively. Clinical data were also obtained.

Results. High counts of CD68+ and CD163+ macrophages were associated with hr-HPV infection (both $p<0.05$ ) and positively correlated with cervical carcinogenesis (Spearman's rho $=0.478$, $p=0.000$; Spearman's rho $=0.676, p=0.000$, respectively). The immunostaining pattern of CD163 exhibited clearer background than that of CD68. CD163+ macrophages showed a more obviously increasing migration into the epithelium along with the progression of $\mathrm{CIN}$ to invasive cancer. Notably, a high index of CD163+ macrophages was significantly associated with higher FIGO stages $(p=0.009)$ and lymph node metastasis $(p=0.012)$, but a similar finding was not found for CD68+ macrophages ( $p=0.067, p=0.079$, respectively).

Conclusions. Our study supported a critical role of TAMs as a prospective predictor for hr-HPV-related cervical carcinogenesis. CD163, as a promising TAMs marker, is superior to CD68 for predicting the malignant transformation and metastatic potential of cervical cancer.
\end{abstract}

Key words: CD68, CD163, cervical cancer, human papillomavirus (HPV), tumor-associated macrophages (TAMs), tumor microenvironment (TME)

\section{Introduction}

Cervical carcinogenesis is a consequence of persistent infection with high-risk human papillomaviruses (hr-HPV) [1]. In recent years, convincing data have emerged that a 
tumor-associated immune microenvironment must be an important determinant of susceptibility to HPV-related cervical cancer [2]. Invasion and metastasis are the primary causes of treatment failure and subsequent death in cervical cancer patients. Moreover, metastasis to lymphatic vessels indicates an unfavorable prognosis [3, 4]. Malignant tumors are complex structures that must interact with the surrounding environment for growth and invasiveness. However, the molecular alterations in the tumor-associated immune microenvironment that drive invasion and metastasis in cervical cancer are not well established. These observations underscore the urgent need to identify new biomarkers with the potential to predict tumor progression, enable diagnosis at earlier stages of the disease, and facilitate the rapid detection of disease recurrence or metastasis following treatment.

Macrophages are critical immune effector cells and one of the major components of tumor-infiltrating leukocytes. These cells play a key role, in terms of their increased levels and their function, in the processes associated with carcinogenesis [5]. Macrophages are derived from blood monocytes and can polarize into M1 (classically activated) or M2 (alternatively activated) subpopulations within the tumor microenvironment (TME) [6, 7]. Accordingly, macrophages that infiltrate and surround the tumor nest are defined as tumor-associated macrophages (TAMs) [8]. TAMs can interact with neoplastic cells by releasing various cytokines that can contribute to cancer initiation and progression. Emerging data suggest that high numbers of TAMs in various types of carcinomas are associated with a poor prognosis [9-11].

Tumor-associated viruses lead to malignancies with a prolonged latency and in conjunction with TME. One of the hallmarks of oncogenic virus-related inflammation is the recruitment of monocyte-macrophage lineage cells to the TME. Several reports suggest that viral infection promotes macrophage activation and M2 polarization [12, 13]. In recent years, strong evidence has emerged that TAMs play a critical role in the induction of virus-associated malignant tumors [14-16]; this highlights the necessity to further explore the influence of TAMs on HPV-related cervical carcinogenesis.

There are several known functional markers of TAMs, and the presence of CD163 versus CD68 is a key distinguishing factor between different TAMs. CD163, a member of the scavenger receptor cysteine-rich family, is involved in anti-inflammatory functions and is predominantly expressed on M2 macrophages [17]. Accumulating evidence indicates that a high number of TAMs, as demonstrated by exclusive immunohistochemistry (IHC) staining with antibodies against CD163, is associated with an unfavorable prognosis in a variety of malignancies [18-20]. However, CD68, the well-established generic macrophage marker, could not distinguish M1 or M2 subtypes from all the infiltrated macrophages [21]. On the basis of these reports, we hypothesized that CD163+ macrophages might be a subpopulation of CD68+ macrophages and are more suitable to identify the tumor-promoting macrophages.

In this study, the distribution and clinical significance of macrophages in cervical squamous dysplasia and carcinoma were evaluated with the markers of CD163 and CD68, and the association between CD163+ macrophages/CD68+ macrophages and hr-HPV infection was analyzed. We also studied the differences between these two markers.

\section{Materials and methods}

\section{Tissue samples}

Between January 2011 and December 2013, 130 archival formalin-fixed paraffin-embedded cervical specimens were obtained from the Department of Obstetrics and Gynecology, Nanfang Hospital, Southern Medical University. The specimens included 26 cases of normal cervical tissues, 30 cases of cervical intraepithelial neoplasia (CIN) I, 29 cases of CIN II-III, and 45 cases of squamous cell carcinoma (SCC) (range from stage I to II). Specimens of lesions were selected consecutively from cervical biopsies (8 cases), loop electrosurgical excision procedure specimens (22 cases), cone biopsies (29 cases), and radical hysterectomy and pelvic lymphadenectomy (45 cases). Twenty-six cases of normal cervical tissues were obtained from patients undergoing hysterectomy for benign conditions without a history of CIN or abnormal Pap smears. All the 45 cases of cervical cancer were submitted for pathological examination after operation. Pregnant women and patients with acute pelvic infection were excluded from the study. Patients in whom adenocarcinoma was diagnosed were excluded. Patients who had received cervical therapy or preoperative treatment, such as radiotherapy and chemotherapy, were also excluded. As outlined in Table 1, HPV test was performed using the hybrid capture II (HC-II, Digene). The cervical specimens were divided into four histological groups: normal, CIN I, CIN II-III, SCC. Clinical pathologic characteristics of the tumor (e.g. age, FIGO stage, histology, lymph node status) were provided by the Department of Obstetrics and Gynecology and Department of Pathology, Nanfang Hospital, Southern Medical University. Informed 
consent was obtained from each patient before using the samples. Serial sections were prepared for hematoxylin and eosin staining and IHC analysis. Two experienced pathologists who had no prior knowledge of the patient data reviewed the slides for all cases to confirm the previous diagnosis independently.

Table 1. Correlation of CD68 and CD163 expression with hr-HPV infection

\begin{tabular}{lllllll}
\hline Group & hr-HPV & CD68 & \multicolumn{3}{l}{ CD163 } & Total \\
\cline { 3 - 5 } & & $\mathrm{H}$ & $\mathrm{L}$ & $\mathrm{H}$ & $\mathrm{L}$ & \\
\hline Normal & $\mathrm{P}$ & $2(7.7)$ & $1(3.8)$ & $3(11.6)$ & $0(0)$ & 3 \\
& $\mathrm{~N}$ & $10(38.5)$ & $13(50)$ & $6(23)$ & $17(65.4)$ & 23 \\
CIN I & $\mathrm{P}$ & $15(50)$ & $6(20)$ & $17(56.7)$ & $4(13.3)$ & 21 \\
& $\mathrm{~N}$ & $5(16.7)$ & $4(13.3)$ & $3(10)$ & $6(20)$ & 9 \\
CIN II-III & $\mathrm{P}$ & $23(79.3)$ & $4(13.8)$ & $23(79.3)$ & $4(13.8)$ & 27 \\
& $\mathrm{~N}$ & $0(0)$ & $2(6.9)$ & $0(0)$ & $2(6.9)$ & 2 \\
SCC & $\mathrm{P}$ & $37(82.2)$ & $8(17.8)$ & $40(88.9)$ & $5(11.1)$ & 45 \\
& $\mathrm{~N}$ & $0(0)$ & $0(0)$ & $0(0)$ & $0(0)$ & 0 \\
Total & & 92 & 38 & 92 & 38 & \\
\hline
\end{tabular}

*Data are given as number (percentage). Fisher Exact Test, $P<0.05$.

$\mathrm{CIN}=$ cervical intraepithelial neoplasia; $\mathrm{SCC}=$ squamous cell carcinoma; $\mathrm{hr}-\mathrm{HPV}=$ high-risk human papillomavirus; $\mathrm{H}=$ high expression; $\mathrm{L}=$ low expression; $\mathrm{N}=$ negative; $\mathrm{P}=$ positive

\section{Immunohistochemistry}

IHC was performed as previously described [22, $23]$. Briefly, $4-\mu \mathrm{m}$-thick consecutive paraffin sections were deparaffinized and rehydrated. Endogenous peroxidase activity was blocked by incubating for 30 min in $3 \% \mathrm{H}_{2} \mathrm{O}_{2}$ in methanol at room temperature. Antigen retrieval was performed using a microwave treatment for $15 \mathrm{~min}$ in citrate buffer $(\mathrm{pH} \mathrm{6.0)}$. The sections were then blocked with $10 \%$ goat serum at $37^{\circ} \mathrm{C}$ for $1 \mathrm{~h}$ and incubated with mouse monoclonal anti-human primary antibodies against CD163 or CD68 (both applied at 1:200, Abcam, USA) in a humidified chamber overnight at $4^{\circ} \mathrm{C}$. Next, the sections were incubated with secondary antibody and processed with the universal SP histostain ${ }^{\mathrm{TM}_{-}}$-plus kit (ZYMED, USA). Finally, the slides were counterstained with hematoxylin.

\section{Evaluation of Immunohistochemistry}

Macrophage infiltration was quantitatively estimated in three separate compartments: the epithelium, the stroma, and both compartments combined. Each specimen was screened at low magnification $(\times 100)$, and the five areas with greatest number of positively stained cells (hot spot area) were selected for further analysis. The mean macrophage count in these five areas for each case was estimated $\left(\right.$ per $\left.\mathrm{mm}^{2}\right)$ at high power $(\times 400)$ magnification. The percentage of immunoreactivity was scored from 0 to 3 according to the proportion of positively stained cells as follows: $0,<5 \% ; 1,5 \%-20 \% ; 2,20 \%-50 \% ; 3$, $>50 \%$. A score of 2 to 3 was classified as high-level expression and 0 to 1 as low-level expression. Mean macrophage count was determined by two independent pathologists who were blinded to the patient's pathological and clinical status. To confirm the reproducibility, $25 \%$ of the slides were chosen randomly and scored twice. All duplicates were evaluated in a similar manner.

\section{Statistical Methods}

Statistical data were analyzed was performed using the IBM Statistical Package for Social Sciences for Windows (version 20.0, IBM, Armonk, NY, USA). $\mathrm{T}$ test and one-way ANOVA were used to determine the statistical significance of the differences between the two macrophage populations (CD163+ and CD68+). Frequency tables were analyzed using the Fisher Exact Test, with Spearman and Pearson Correlation Coefficient used to assess the significance of correlations between categorical variables. Values with distribution are presented as mean \pm standard deviation. All tests were two-tailed, and $p<0.05$ was considered statistically significant.

\section{Results}

\section{Upregulation of CD68+ macrophages is correlated with cervical malignant transformation}

CD68 was localized within the cytoplasm of the macrophages and exhibited granular, brownish staining in cervical specimens (Figure 1A). The progression from the normal cervical epithelium to dysplasia and SCC was associated with a progressive increase in the immunoreactivity of CD68+ macrophages (100× magnification) (Figure 1C).

The expression levels of CD68+ macrophages in the four histological groups, i.e, normal cervical tissue, CINI, CINII-III, and SCC, were analyzed. Statistical analysis revealed that the levels of total CD68+ macrophages in SCC tissues were significantly higher than those in the normal cervical tissue $(p<$ $0.05)$, CINI $(p<0.05)$, and CIN II-III $(p<0.05)$. In addition, an upregulation of CD68+ macrophages was observed in CIN II-III compared with that in CIN I ( $p$ $<0.05)$ and normal tissues $(p<0.05)$. The number of total $\mathrm{CD} 68+$ macrophages in tissues of increasing histological grade, from normal cervix to SCC, was 12.5, 46.2, 93.5, and 178.7 (Figure 2A), respectively. A positive association between $\mathrm{CD} 68+$ macrophage expression and cervical carcinogenesis and carcinoma progression was observed when we analyzed the immunoreactivity of CD68 (Spearman's rho $=0.478, p$ $=0.000)$ (Figure 2B). 


\section{Upregulation of CD163+ macrophages is correlated with cervical malignant transformation}

CD163 immunoreactivity is characterized by a granular brownish pattern and membrane staining in cervical specimens (Figure 1B). CD163 staining pattern exhibited a cleaner background than CD68 with less nonspecific staining of carcinoma cells and other inflammatory elements (Figure 1A-1B). The more advanced the lesions of the cervix, the higher was the immunoreactivity of CD163+ macrophages (100× magnification) (Figure 1C).

We also observed a progressive increase in total CD163+ macrophages with increasing histological grade (from normal tissue to SCC), with cell counts ranging from a median value of 8.3 in normal tissues to 145.7 in SCC (Figure 2C). The expression level of CD163 was significantly higher in SCC than in different CINs (all $p<0.05$ ), and the CD163 expression levels in different CINs were also higher than that in normal cervix (all $p<0.05$ ). In addition, the difference was statistically significant between the early and late stages of CIN $(p<0.05)$. The density of CD163+

A

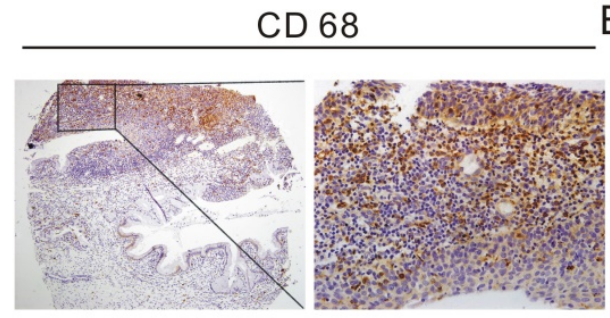

C
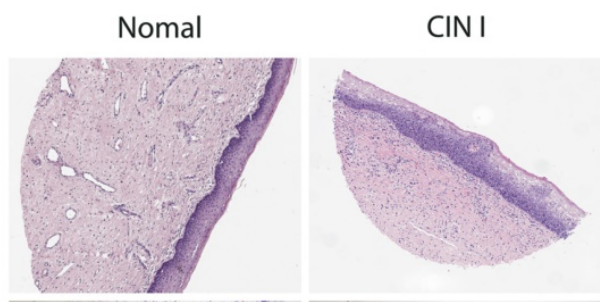

B
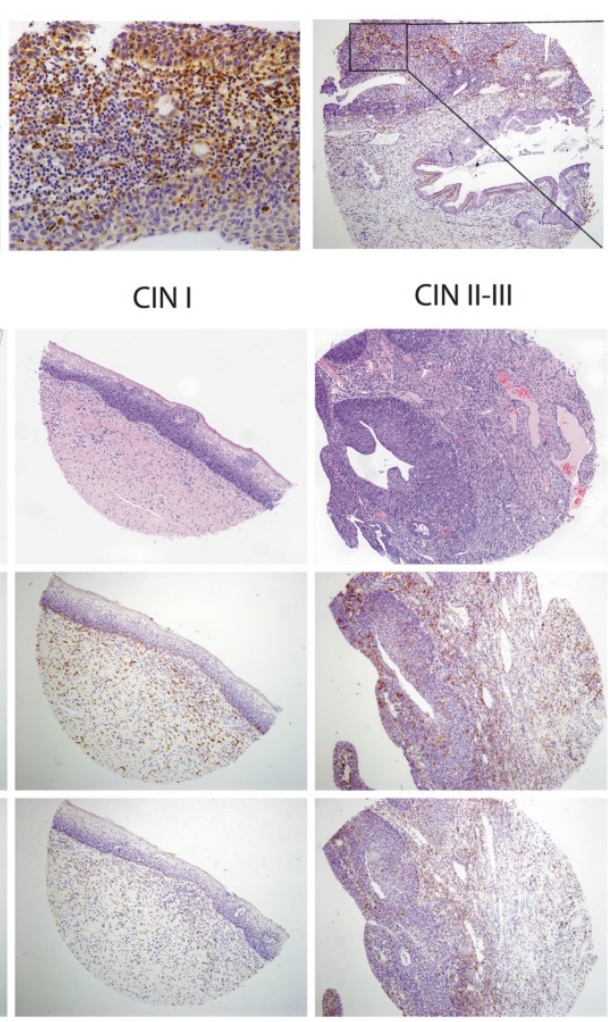

Figure 1. Immunohistochemical detection of CD68+ and CD163+ macrophage expression in different cervical tissues. Representative immunostaining of CD68 (A) and CD163 (B) on consecutive sections of paired cervical tissue (magnification $\times 100$ left and $\times 400$ right). (C) Normal cervical tissue showed weak positive staining with scattered CD68+ and CD163+ macrophages. Cervical intraepithelial neoplasia (CIN) I displayed weak positive staining with few CD68+ and CD163+ macrophages. CIN II-III showed strong immunohistochemical staining with the increase in CD68+ and CD163+ macrophage population. Squamous cell carcinoma (SCC) showed intense staining and high count of CD68+ and CD163+ macrophages. macrophages demonstrated a positive correlation with the progression of cervical carcinogenesis (Spearman's rho $=0.676, p=0.000)$ (Figure 2D).

\section{Expression of both CD68+ and CD163+ macrophages is related to hr-HPV infection}

The relationship between the expression of either CD68+ or CD163+ macrophages and hr-HPV infection in cervical lesions was assessed. Results of hr-HPV infection rates were available for 26 normal, 30 CIN I, 29 CIN II-III, and 45 SCC cases at $11.5 \%$, $70 \%, 93.1 \%$, and $100 \%$, respectively.

The density of both CD163+ and CD68+ macrophage counts was significantly greater in the hr-HPV-positive group than in the hr-HPV-negative group (all $p<0.05$ ) (Figure 3A). As shown in Table 1, there was a significant correlation between the different grades of CD163+ and the hr-HPV infection status in different histological cervical lesions: normal, CIN I, CIN II-III, SCC (all $p<0.05$ ). The expression of CD68+ macrophages was significantly correlated with hr-HPV infection in cervical lesions of CIN II-III and SCC (both $p<0.05$ ). In all the cases, high expression levels of CD163+ and CD68+ macrophages were more frequent in hr-HPV-positive lesions than those in hr-HPV-negative

lesions, of which $55.9 \%$ had low expression for CD68 (19 of 34) and $73.5 \%$ for CD163 (25 of 34). In the hr-HPV-positive cases, $80.2 \%$ (77 of 96) showed high expression of CD68, as compared to $44.1 \%$ (15 of 34 ) of the hr-HPV-negative cases. High levels of CD163+ macrophage expression in all cases infected with hr-HPV was significantly higher (11.6\% for normal, $56.7 \%$ for CIN I, $79.3 \%$ for CIN II-III, $88.9 \%$ for SCC) than those without hr-HPV infection $(23 \%$ for normal, $10 \%$ for CIN I, $0 \%$ for CIN II-III, $0 \%$ for SCC). 

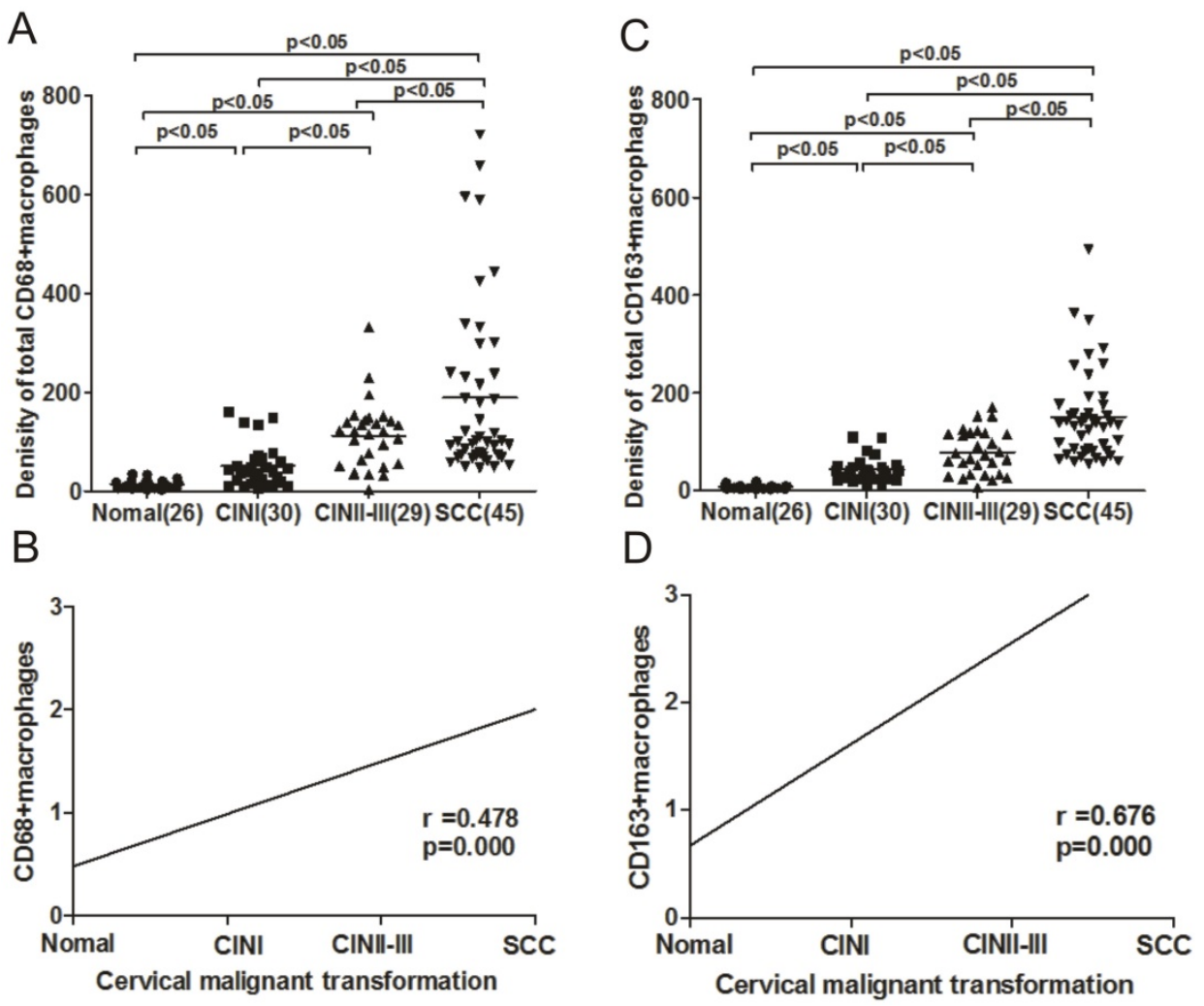

Figure.2. Upregulation of both CD68+ and CD163+ macrophages is correlated with cervical carcinogenesis and carcinoma progression. The infiltration density of $\mathrm{CD} 68+(\mathrm{A})$ and $\mathrm{CDI} 63+$ macrophages $(\mathrm{C})$ in normal cervical tissue, cervical intraepithelial neoplasia $(\mathrm{CIN}) \mathrm{I}$, CIN II-III, and squamous cell carcinoma (SCC). Bars indicate median immunostaining score values. The level of CD68+ (B) and CD163+ (D) macrophage expression showed a positive correlation with cervical malignant transformation $(r=0.487, p=0.000 ; r=0.676, p=0.000$; Spearman correlation coefficient test). The line represents the calculated regression line.

A

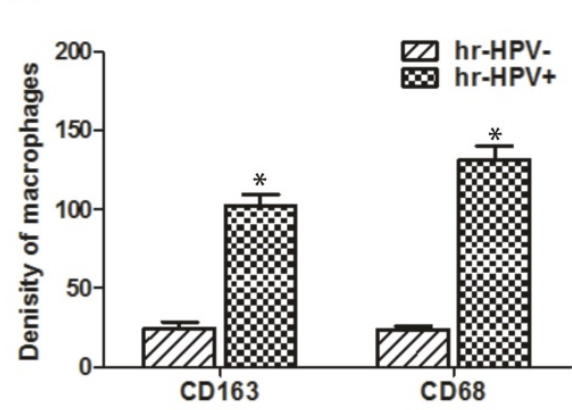

C

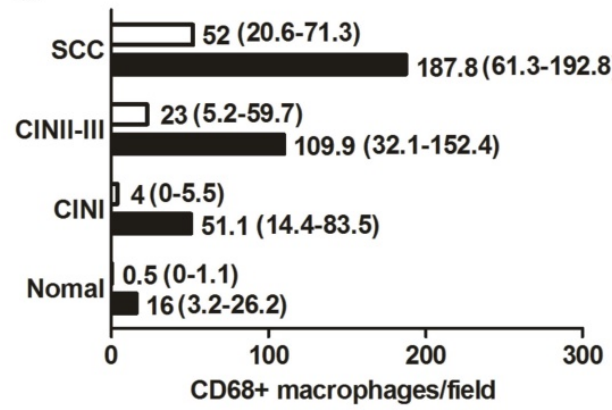

B
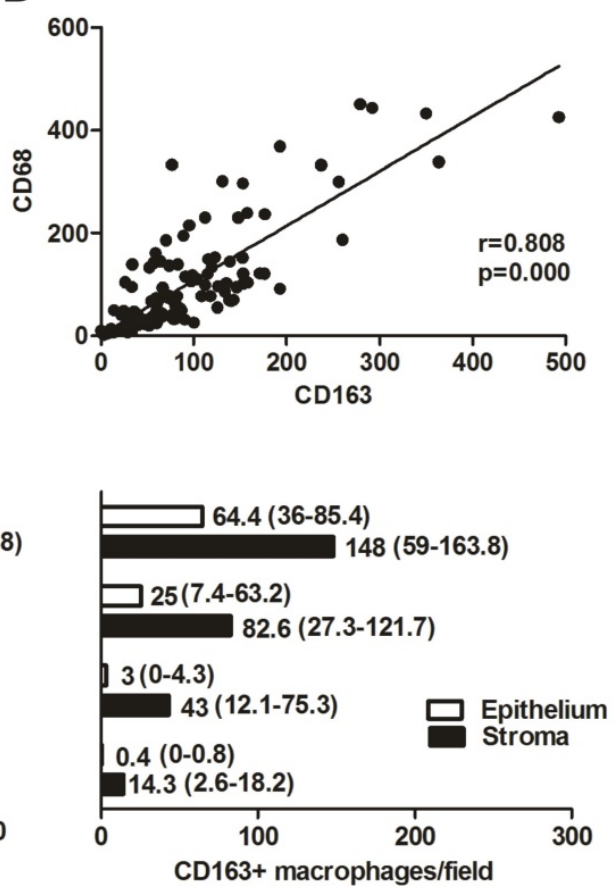

Figure 3. The correlation between CD68+ and CD163+ macrophages during cervical carcinogenesis and carcinoma progression. (A) Significant differences in the mean macrophage counts between the subgroups with or without high-risk human papillomavirus (hr-HPV) infection (all $p<0.05$ ). (B) Correlation of macrophages measured by CD68 or CD163 in different histological cervical lesions. The level of CD68+ macrophages showed a positive correlation with CD163+ macrophages $(r=0.808, p=0.000$, Pearson Correlation Coefficient). The line represents the calculated regression line. (C) The counts of CD68+ and CD163+ macrophages (per $\times 400$ field) in epithelium (white bars) and stroma (black bars) in different histological cervical lesions: normal, cervical intraepithelial neoplasia (CIN) I, CIN II-III, squamous cell carcinoma (SCC). Values presented in median (25th-75th percentile). 


\section{CD163+ macrophages tend to correlate with malignant transformation of the cervix more obviously than CD68+ macrophages}

Although the average number of CD68+ macrophages was higher than that of CD163+ macrophages, the total macrophage counts revealed that the number of CD68+ macrophages was directly correlated with the number of CD163+ macrophages (Pearson Correlation $=0.808, p=0.000$ ) (Figure 3B). We also studied CD163+ macrophage/CD68+ macrophage infiltration in either stroma or epithelium of the four histological groups respectively (Figure 3C). The stromal compartment consistently demonstrated higher counts than the epithelium for both CD163+ and CD68+ macrophages. The stroma/epithelium ratio of $\mathrm{CD} 163+$ and $\mathrm{CD} 68+$ macrophages significantly decreased as the lesions progressed, indicating a macrophage switch from the stroma to the epithelium, which was more obvious for CD163+ macrophages.

\section{CD163+ macrophages tend to correlate with FIGO stage and lymph node metastasis of cervical cancer more closely than CD68+ macrophages.}

To investigate the clinical significance of CD163+ and CD68+ macrophages levels in cervical carcinoma, we evaluated the correlation between the levels of CD163+ macrophages/CD68+ macrophages and the clinicopathological features in serial sections of the same tissues. As shown in Table 2, CD163+ and CD68+ macrophages were not significantly correlated with age or histologic type (all $p>0.05$ ). The counts of total CD163+ macrophages were significantly increased in cases presenting with lymph node metastasis and higher FIGO stages $(p=0.012, p=$ 0.009 , respectively); however, the same results were not observed for CD68+ macrophages $(p=0.079, p=$ 0.067, respectively).

Previous studies have suggested that late FIGO stages and lymph node metastasis are the primary causes of treatment failure and subsequent death in patients with cervical cancer $[3,4]$. Thus, we further explored the correlation between macrophage expression and FIGO stage/lymph node metastasis. As shown in Table 3, increasing CD163+ macrophage counts correlated with a more advanced tumor stage (Spearman's rho $=0.502, p=0.020$ ). However, there was no significant difference between CD68+ macrophage expression and FIGO stage (Spearman's rho $=0.389, p=0.137)$. In addition, CD163+ macrophage expression was more significantly correlated with lymph node metastasis than CD68+ macrophage expression (Spearman's rho $=0.637, p=$ 0.005 ; Spearman's rho $=0.407, p=0.085$, respectively).

Table 2. Correlation of increased CD68 and CD163 expression with clinicopathologic features

\begin{tabular}{|c|c|c|c|c|c|}
\hline \multirow[t]{2}{*}{ Variable } & \multirow[t]{2}{*}{ No. } & \multicolumn{2}{|c|}{$\begin{array}{l}\text { Density of total CD163+ } \\
\text { macrophage cell number }\end{array}$} & \multicolumn{2}{|c|}{$\begin{array}{l}\text { Density of total CD68+ } \\
\text { macrophage cell } \\
\text { number }\end{array}$} \\
\hline & & $\mathrm{X} \pm \mathrm{SD}$ & $\mathrm{P}$ & $\mathrm{X} \pm \mathrm{SD}$ & $\mathrm{P}$ \\
\hline \multicolumn{6}{|l|}{ Age } \\
\hline$<45$ years & 19 & $120.75 \pm 40.8$ & 0.263 & $137.57 \pm 41.3$ & 0.310 \\
\hline$\geq 45$ years & 26 & $117.59 \pm 46.6$ & & $132.92 \pm 45.9$ & \\
\hline \multicolumn{6}{|c|}{ FIGO stage } \\
\hline I & 21 & $104.60 \pm 34.8$ & $0.009^{*}$ & $130.26 \pm 34.3$ & 0.067 \\
\hline II & 24 & $133.71 \pm 40.3$ & & $140.21 \pm 42.8$ & \\
\hline \multicolumn{6}{|l|}{ Histology } \\
\hline High & 13 & $123.74 \pm 42.1$ & 0.573 & $138.70 \pm 31.9$ & 0.650 \\
\hline Middle & 20 & $115.60 \pm 46.3$ & & $129.89 \pm 41.4$ & \\
\hline Low & 12 & $118.40 \pm 32.6$ & & $136.90 \pm 42.8$ & \\
\hline \multicolumn{6}{|l|}{$\begin{array}{l}\text { LN } \\
\text { metastasis }\end{array}$} \\
\hline Negative & 18 & $100.02 \pm 31.2$ & $0.012^{*}$ & $126.41 \pm 37.6$ & 0.079 \\
\hline Positive & 27 & $138.40 \pm 45.5$ & & $144.13 \pm 45.8$ & \\
\hline
\end{tabular}

*Statistical significance $(P<0.05)$ was calculated using the $t$ test and one-way ANOVA.

FIGO = International Federation of Gynecology and Obstetrics; LN = lymph node.

Table 3. Spearman correlation coefficient of increased CD68 and CD163 expression with clinicopathologic features

\begin{tabular}{lllll}
\hline Variable & \multicolumn{2}{l}{ CD163+ macrophages } & \multicolumn{2}{l}{ CD68+ macrophages } \\
\cline { 2 - 5 } & $\begin{array}{l}\text { Spearman's } \\
\text { rho }\end{array}$ & $\begin{array}{l}\text { Spearman's } \\
\text { rho }\end{array}$ & $\mathrm{p}$ \\
\hline FIGO stage & 0.502 & $0.020^{*}$ & 0.389 & 0.137 \\
LN metastasis & 0.637 & $0.005^{*}$ & 0.407 & 0.085 \\
*Statistical significance $(P<0.05)$ was calculated using the Spearman correlation \\
coefficient. \\
FIGO = International Federation of Gynecology and Obstetrics; LN = Lymph node.
\end{tabular}

\section{Discussion}

HPV-related cervical carcinogenesis is a complex structure that relies on interactions with the surrounding environment to promote their growth and invasive capabilities. Chronic inflammation of virus-associated malignant tumors is characterized by the infiltration of various leukocyte populations including activated macrophages [24]. Accumulating evidence suggests that a high number of TAMs in TME is strongly associated with viral infection [14-16]. Our findings suggest a critical role for both CD163+ and CD68+ macrophages in HPV infection and demonstrate a strong association between the malignant transformation of the cervix and an increase in the number of CD163+ and CD68+ macrophages. These data support a role of TAMs as a prospective predictor for HPV-related cervical carcinogenesis, which highlight the importance for a further study of TAMs involved in cervical cancer.

We further investigated the specific distribution of both CD163+ and CD68+ macrophages in cervical 
cancer tissue. In most of the samples that we evaluated, both CD163+ and CD68+ macrophages localized in the same tissue regions. However, CD163 showed lower staining levels than CD68, with less nonspecific staining of background inflammatory cells or carcinoma cells. CD68 immunostaining can be observed in multiple cell types, such as fibroblasts and inflammatory cells. In the case of cervical carcinoma, there are myriad of inflammatory and stromal elements surrounding the relatively few neoplastic cells, and high background staining can complicate the results when using the nonspecific CD68 marker. CD163 appears to be a more accurate marker for identifying TAMs in cervical carcinoma.

Currently, accumulating evidence indicates that the interaction of the tumor stroma, a key component of the TME, with cancer cells might facilitate the process of tumor progression [25]. In our study, the stromal compartment consistently demonstrated higher macrophage counts than the epithelium. We also found that increased macrophage counts in the epithelium were directly correlated with malignant transformation in cervical cancer and that this correlation was particularly pronounced for CD163+ macrophages. Therefore, we presume that tumor cells in the TME secrete various types of cytokines to promote the migration of monocytes to the tumor tissue and that macrophages infiltrating the nests can directly interact with the tumor cells. Moreover, this finding highlights the crucial role of CD163+ macrophages. As opposed to $\mathrm{CD} 68+$ macrophage infiltration, a high level of CD163+ macrophage infiltration was more strongly associated with malignant transformation in cervical cancer.

Interestingly, regarding the patient clinicopathological data, a high CD163+ macrophage count was significantly associated with advanced FIGO stage and lymph node metastasis. A potential mechanism to explain the clinical relevance of these findings is that, upon activation by tumors, macrophages adopt a phenotype associated with poor antigen-presenting capability, suppression of $\mathrm{T}$ cell proliferation, increased angiogenesis, and increasing tissue remodeling capability. Together, these effects facilitate tumor cell invasion and metastasis [26]. Because advanced disease stage and lymph node metastasis is the major cause for cervical cancer-related deaths, we considered that CD163+ macrophages have a clinical significance as a prognostic indicator in patients with cervical cancer. An association between the number of CD68+ macrophages and lymph node metastases was identified by Ding and Dongju et al. [27, 28]. Other groups, in accordance with our observation, have not been able to reproduce these results [29]. Our study (in which lesions IHC data were compared with clinicopathological data) reported a positive association between infiltrating CD163+ macrophages and cervical carcinogenesis and carcinoma progression; this finding will be valuable to further research investigating the significance of TAMs in cervical cancer.

There is an increasing body of evidence suggesting that increased TAMs infiltration in human tumors is associated with a poor prognosis [9-11, 18-20]. Interestingly, some researchers argued that the level of CD163+ macrophages is a better prognostic indicator than CD68 [30-32]. However, there are few reports on the role of CD163+ and CD68+ macrophages in cervical carcinoma, and data regarding their prognostic relevance are limited and disputable. Carus et al. studied 101 FIGO stage IB and IIA cervical cancer patients and found that the peritumoral CD163+ macrophage count was significantly associated with reduced recurrence-free survival (RFS) [33]. In HPV-related cervical cancer, the intraepithelial infiltration with high numbers of M1 macrophages is an independent prognostic factor for a favorable survival [34]. Nedergaard et al. reported that no significant association between CD68+ macrophages and tumor recurrence [35]. The current study demonstrates that the capability of macrophage counts, as assessed by IHC, to predict the prognosis of cervical cancer patients needs to be further explored.

In conclusion, our study clearly demonstrates a positive association between the expression of either CD68+ or CD163+ macrophages and cervical carcinogenesis. The number of macrophage increase linearly with the infection of hr-HPV. The level of CD163+ macrophages exhibited a stronger correlation with the advanced FIGO stage and lymph node metastasis of cervical cancer than CD68+ macrophages. Additionally, CD163 exhibited cleaner background immunostaining than CD68. These results suggest that TAMs might offer a novel approach for cervical cancer treatment, and in upcoming trials, CD163+ macrophages should be investigated as a potential marker of disease progression. However, because of our limited sample size, we were unable to evaluate the prognostic capability of CD163+ and CD68+ macrophages in cervical cancer. Additional studies with larger sample sizes and prospective cases are required to verify the significance of macrophage infiltration as a prognostic marker in cervical carcinoma. Furthermore, our study should promote further exploration on the mechanism by which HPV and TAMs cooperate in the tumor microenvironment and how this cooperation influences cervical carcinogenesis and metastasis. 


\section{Acknowledgments}

This work was supported by the National Science Foundation of China (No. 81372781; 81072132; 81572546; 81672589); the Natural Science Foundation of Shanghai Municipality (No.15ZR1433300); and the Science and Technology Program of Shenzhen (No. JCYJ20160429161218745).

\section{Competing Interests}

The authors have declared that no competing interest exists.

\section{References}

[1] Arbyn M, Castellsague X, de Sanjose S, et al. Worldwide burden of cervical cancer in 2008. Ann Oncol. 2011; 22 (12) : 2675-2686.

[2] Deligeoroglou E, Giannouli A, Athanasopoulos N, et al. HPV infection: immunological aspects and their utility in future therapy. Infect Dis ObstetGynecol. 2013; 2013: 540850.

[3] Colombo N, Carinelli S, Colombo A, et al. Cervical cancer: ESMO Clinical Practice Guidelines for diagnosis, treatment and follow-up. Ann Oncol. 2012; 23(Suppl 7): S27-S32.

[4] Lee MY, Shen MR. Epithelial-mesenchymal transition in cervical cancer. Am J Transl Res. 2012; 4: 1-13.

[5] Condeelis J, Pollard JW. Macrophages obligate partners for tumor cell migration, invasion, and metastasis. Cell. 2006; 124 (2) : 263-266.

[6] Lewis CE, Pollard JW. Distinct role of macrophages in different tumor microenvironments. Cancer Res. 2006; 66 (2) : 605-612.

[7] Martinez FO, Helming L, Gordon S. Alternative activation of macrophages: an immunologic functional perspective. Annu Rev Immunol. 2009; 27: 451-483.

[8] Wang YC, He F, Feng F, et al. Notch signaling determines the M1 versus M2 polarization of macrophages in antitumor immune responses. Cancer Res. 2010; 70 (12) : 4840-4849.

[9] Medrek C, Ponten F, Jirstrom K, et al. The presence of tumor associated macrophages in tumor stroma as a prognostic marker for breast cancer patients. BMC Cancer. 2012; 12: 306

[10] Steidl C, Lee T, Shah SP, et al: Tumor-associated macrophages and survival in classic Hodgkin's lymphoma. The New England journal of medicine. 2010; 362 (10) : 875-885

[11] Ruffell B, Coussens LM. Macrophages and therapeutic resistance in cancer. Cancer Cell. 2015; 27 (4) : 462-72.

[12] Bility MT, Cheng L, Zhang Z, et al. Hepatitis B virus infection and immunopathogen esis in a humanized mouse model: induction of human-specificliver fibrosisinduction of human-specific liver fibrosis and M2-like macrophages. PloS Pathog. 2014; 10(3): e1004032.

[13] Burdo TH, Walker J, Williams KC. Macrophage Polarization in AIDS: Dynamic Interface between Anti-Viral and Anti-Inflammatory Macrophages during Acute and Chronic Infection. J Clin Cell Immunol. 2015; 6 (3).

[14] Tan KL, Scott DW, Hong F, et al: Tumor-associated macrophages predict inferior outcomes in classic Hodgkin lymphoma: a correlative study from the E2496 Intergroup trial. Blood. 2012; 120 (16) : 3280-3287.

[15] Shi B, Ren G, Hu Y, et al. HBsAg inhibits IFN-alpha production in plasmacytoid dendritic cells through TNF-alpha and IL-10 induction in monocytes. PLoS One. 2012; 7 (9) : e44900.

[16] Wang S, Chen Z, Hu C, et al. Hepatitis B virus surface antigen selectively inhibits TLR2 ligand-induced IL-12 production in monocytes/macrophages by interfering with JNK activation. J Immunol. 2013; 190 (10) : 5142-5151.

[17] Nguyen TT, Schwartz EJ, West RB, et al. Expression of CD163 (hemoglobin scavenger receptor) in normal tissues, lymphomas, carcinomas, and sarcomas is largely restricted to the monocyte/macrophage lineage. Am J SurgPathol. $2005 ; 29$ (5) : 617-624.

[18] Jensen TO, Schmidt H, Moller HJ, et al. Macrophage markers in serum and tumor have prognostic impact in American Joint Committee on Cancer stage I/II melanoma. J ClinOncol. 2009; 27 (20) : 3330-3337.

[19] Komohara Y, Hasita H, Ohnishi K, et al. Macrophage infiltration and its prognostic relevance in clear cell renal cell carcinoma. Cancer science. 2011; 102 (7) : 1424-1431

[20] Lima L, Oliveira D, Tavares A, et al. The predominance of M2-polarized macrophages in the stroma of low-hypoxic bladder tumors is associated with BCG immunotherapy failure. Urologic oncology. 2014; 32 (4) : 449-457.

[21] Falini B, Flenghi L, Pileri S, et al. PG-M1: a new monocl onal antibody directed against a fixative-resistant epitope on the macrophage-restri cted form of the CD68 molecule. Am J Pathol. 1993; 142: 1359-1372.16.

[22] Wang W, Wang S, Song $X$, et al. The relationship between c-FLIP expression and human papillomavirus E2 ge ne disruption incervical carcinogenesis. Gynecol Oncol. 2007; 105(3): 571-7.
[23] Li $Y$, Wang $W$, Wang $W$, et al Correlation of TWIST2 up-regulation and epithelial-menchymal transition during tumorigenesis and progression of cervical carcinoma. Gynecol Oncol. 2012; 124(1): 112-8.

[24] Mantovani A, Romero P, Palucka AK, et al. Tumour immunity: effector response to tumour and role of the microenvironment. Lancet .2008; 371 (9614) : 771-783.

[25] Bissell MJ, Radisky D. Putting tumours in context. Nat Rev Cancer. 2001; 1 (1) : 46-54.

[26] Pollard JW. Tumour-educated macrophages promote tumour progression and metastasis. Nat Rev Cancer. 2004; 4 (1) : 71-78.

[27] Ding H, Cai J, Mao M, et al. Tumor-associated macrophages induce lymphangiogenesis in cervical cancer via interaction with tumor cells. Apmis. 2014; 122 (11) : 1059-1069.

[28] Liu Dongiu, Lou Ge. The relationship between macrophages infiltration and VEGFR-3 positive vessel density in uterine cervical cancers. Chinese Journal of Clinical Oncology. 2005; 32 (4) : 189-191.

[29] Davidson B, Goldberg I, Gotlieb WH, et al. Macrophage infiltration and angiogenesis in cervical squamous cell carcinoma--clinicopathologic correlation. Acta Obstet Gynecol Scand. 1999; 78 (3) : 240-244.

[30] Zaki MA, Wada N, Ikeda J, et al: Prognostic implication of types of tumor-associated macrophages in Hodgkin lymphoma. Virchows Arch. 2011; 459 (4) : 361-366

[31] Barros MH, Hassan R, Niedobitek G. Tumor-associated macrophages in pediatric classical Hodgkin lymphoma: association with Epstein-Barr virus, ymphocyte subsets, and prognostic impact. Clin Cancer Res. 2012; 18 (14) : 3762-3771.

[32] Klein JL, Nguyen TT, Bien-Willner GA, et al. CD163 immunohistochemistry is superior to CD68 in predicting outcome in classical Hodgkin lymphoma. Am J ClinPathol. 2014; 141 (3) : 381-387.

[33] Carus A, Ladekarl M, Hager H, et al. Tumour-associated CD66b+ neutrophil count is an independent prognostic factor for recurrence in localised cervical cancer. Br J Cancer. 2013; 108 (10) : 2116-2122.

[34] de Vos van Steenwijk PJ, Ramwadhdoebe TH, Goedemans R, et al. Tumor-infiltrating CD14-positive myeloid cells and CD8-positive T-cellsprolong survival in patients with cervical carcinoma. Int J Cancer. 2013; 133 (12) : 2884-94.

[35] Nedergaard BS, Nielsen K, Nyengaard JR, et al. Stereologic estimation of the total numbers, the composition and the anatomic distribution of lymphocytes in cone biopsies from patients with stage I squamous cell carcinoma of the cervix uteri. Apmis. 2007; 115 (12) : 1321-1 